\title{
AC 2009-677: REMOTELY RECONFIGURABLE, SECURE WIRELESS MESH NETWORK FOR BIDIRECTIONAL DATA COMMUNICATION
}

\author{
Akram Hossain, Purdue University, Calumet \\ Akram Hossain, Purdue University-Calumet Akram Hossain is a professor in the department of \\ Electrical and Computer Engineering Technology at Purdue University Calumet, Hammond, IN. \\ He worked eight years in industry at various capacities. He is working with Purdue University \\ Calumet for the past 21 years. He consults for industry on process control and related disciplines. \\ $\mathrm{He}$ is a senior member of IEEE. He served in IEEE/Industry Application Society for the past 15 \\ years at various capacities. He served as chair of Manufacturing Systems Development \\ Applications Department of IEEE/IAS. He authored more than 25 refereed journal and \\ conference publications. From 2003 through 2006, he was involved with Argonne National \\ Laboratory, Argonne, IL in developing direct computer control for hydrogen powered \\ automotives. He is also involved in several direct computer control and wireless process control \\ related research projects. His interests are in the area of industrial transducer, industrial process \\ control, wireless controls, statistical process control, computer aided design and fabrication of \\ printed circuit board, programmable logic controllers, programmable logic devices and renewable \\ energy related projects.
}

\section{Vijay Mahajan, Influx Software Solutions}

Education BE (Electronics), India MS ( Tech ), Purdue University Calumet, Hammond, IN

Work Experience 2003 to 2006 as a Project Engineer, Larson and Toubro Responsibility: Preparation of Operation and Maintenance manuals of each product of client.

Currently working: Influx Software Solution as a SAP (HR) functional consultant. 


\title{
REMOTELY RECONFIGURABLE, SECURE WIRELESS \\ MESH NETWORK FOR BIDIRECTIONAL \\ DATA COMMUNICATION
}

\begin{abstract}
In today's Electronic world, there are many devices which connect to one another as well to computer in a variety of ways: A cable connects a computer's processing unit to a display, a data cable and a docking cradle connect a personal digital assistant (PDA) or a cellular phone to a computer, radio waves connect a cordless phone to its base unit, an infrared beam connects a remote control to a television. The elaborate array of connectors among electronic devices cries out for a better solution.

In this fast growing technology world, wireless data transferring in a desired format is essential. Data integrity, data security, cost of hardware and software, and system's power management capacity is of prime importance for our data acquisition application. With the increasing use of data loggers to collect information in industrial and environmental monitoring applications, has come a corresponding increase in the use of wireless technology to retrieve the information. Secure and remotely reconfigurable data acquisition system having several nodes communicating in a bidirectional way is essential for the most industrial/commercial and household applications. Although, improved range of wireless system can be obtained at increased price. Limited range and high cost of available wireless data acquisition system inhibits many potential applications.
\end{abstract}

This project is to develop a low cost, reconfigurable, secure, bidirectional wireless mesh network for industrial/commercial and household applications. A low cost and reconfigurable wireless mesh network will contribute significantly in to currently available communications infrastructure. This wireless mesh network system would provide a solution for customizing a bidirectional sensor network. The wireless mesh network will also provide wireless inventory solution in a large warehouse and could facilitate many other similar applications. This wireless system has features that could be used to transmit requests or commands out the serial port to control or query a desired sensor or an instrument directly. The system shall be fully customizable, network-able and easily configurable.

This type of reconfigurable and secure wireless mesh network system will significantly reduce installation cost, improving data security, data integrity and power consumption. The wireless mesh network system will not only offer potential cost saving, but they can reduce system downtime propel by integrated and programmable power management protocol. This system will enhance both network intelligence and system's overall functional capabilities, as well.

\section{Introduction}

A wireless mesh network (WMN) is defined as a network with nodes having connections to the several other network nodes. In a full mesh network, every node has a connection to every other node. In a partial mesh network, every node isn't connected to every other node; instead, only a 
subset of nodes is joined. Only wireless mesh network system provides the node to node and any node to base station wireless communication. WMN system has the scope to reconfigure any node as well as base station as desired. ${ }^{1}$

Benefits of a mesh networks are: lower costs, scalability, and easy installation. In practice a wireless mesh is preferable over a single-hop wireless network because it can reach beyond tightly controlled environment without increasing the transceiver range. It can adapt to changing conditions and extend beyond single-hop wireless access point. Since full wireless mesh network system provides node to node and any node to base station wireless communication. WMN system has the scope to reconfigure any node as well as base station as desired. Thus intrinsic redundancy is due to its mesh communication protocol.

Unlike wired network, where the signal interference is minimal, wireless network and more specifically mesh networks are susceptible to a few external effects. Such as interference with other RF networks operating in same frequency band. Other limitations are: it can hold single communication at a given time. This means that in a single radio ad hoc mesh network, the best it can do is $(1 / 2)^{\mathrm{n}}$ at each hop. So in a multi hop mesh network, the maximum available bandwidth (Bandwidth means rate of data transfer, throughput or bit rate, measured in bits per second) degrades at the rate of $1 / 2,1 / 4,1 / 8$. Thus by the time it makes four hops it degrades to $1 / 16$ of the total available bandwidth. ${ }^{2}$

This WMN based data acquisition system we have developed, will provide configurability, adaptability, power management capacity, reduced cost, and data integrity. It ensures the safe transmission of data packets to the base station and in turn to computer. A node in the network can send and receive a message, and can also function as a router and relay messages for its neighbor. It must offer multiple redundant communication paths throughout the network. If one link fails for any reason, such as due to node's hardware failure or, introduction of strong RF interference, the network should be able to automatically find another nearby active node and routes messages through alternate paths. Thus WMN remains as a prime candidate for our application and many other industrial and as well as house hold applications.

\section{Research Objectives}

The objective is to design and develop a secure and remotely reconfigurable data acquisition system having several nodes communicating in a bidirectional way for the most industrial and household applications. The ultimate objective of this data acquisition system with multiple nodes is to collect analog and digital data from various wind turbine experiment stations. This reconfigurable multi-node and multi-hop data acquisition system must have remote I/O capability. Currently, high cost of available single-hop wireless data acquisition system inhibits many potential low budget applications.

The purpose of this reconfigurable and secure wireless mesh network system is to significantly reduce installation cost, adoptability to RF interference, improving data security, data integrity and power consumption. We are planning to implement the research objectives by using a generic wireless transceiver module offered by CIRRONET. Wireless mesh network system not only offer potential cost saving, but they can reduce system downtime propelled by integrated 
and programmable power management protocol. This system should enhance both network intelligence and system's overall functional capabilities, as well.

\section{Significance of the Objectives}

The low cost and reconfigurable wireless mesh networks will contribute significantly in to currently available wireless data acquisition and communications infrastructure. Without having a remotely reconfigurable node to node wireless bidirectional communication network in application like ours, limits the ability to acquiring desired data from various devices. Successful design and development of a WMN system having multi-node and multi-hop capabilities will provides the solution to the stated problem.

Frequently between a Wi-Fi device and its base station, the signal strength drops significantly because wireless communication quality degrades with distance due to obstacles and other interferences often irregularly. In practice, these two effects mean that simple, single-hop wireless networks have limited reach. Furthermore, to increase the distance of the data acquiring point, transceiver module power need to be increased and may require FCC licensing. Thus for wireless signal to reach beyond tightly controlled environments, such as office buildings, WMNs offer an attractive solution. They let networks adapt to changing conditions, extend beyond single-hop wireless and wired access points, and has inherent redundancy against failures.

\section{System Hardware and Software}

\section{Module Introduction}

Generic wireless modules, DM2200-916VMs by CIRRONET, have been used to design and develop the proposed mesh networks. This network can be used to monitor and control a wide range of physical parameters, conditions and events. Although the such network could be applied to utility metering and building sub-metering, public works, agriculture, food processing, waste management, manufacturing and industrial processes, security, building automation, and transportation, we have applied the network to collect wind velocity, temperature and humidity data from various wind turbine experiment station located at different physical locations. We also designed the Graphical User Interface (GUI) (A graphical user interface (GUI, is a type of user interface which allows people to interact with electronic devices such as computers; handheld devices such as data acquisition module, gaming devices, household appliances, and office equipment.) using Visual Basic. Using the Object Linking and Embedding (OLE) in Visual Basic we could create excel sheet and store all collected data in it.

Figure 1 shows the arrangement of the node-modules in different floor of Andrey Potter Laboratory Building. Module having Node ID 0055 was placed on the rooftop with the wind and temperature sensor. Module having Node ID 0025 was placed in the staircase area to facilitate the transmission of data from the rooftop node. Module having node ID 0035 was left unconnected to minimize the data retrieval time and limit the number of reading from the rooftop node. Figure 2 shows the portion of the excel data sheet. 
DM2200-916VM System Characteristic Specification [Copyright 2006 - 2007 CIRRONET]

Network Size

Network Creation

Air Data Rate

Transmission Latency

Open Field Range

Routing

Routing Methodology

Network Modes

Node Mobility Support

Messaging Modes

Encryption

Node Power Management

ACK Mechanism
: up to 1500 nodes

: by node binding

: $9600 \mathrm{~b} / \mathrm{s}$

: 260-ms/hop typical

: 600-meters/hop typical

: possible through any node

: rules-based, self-optimizing mesh

: point-to-point, point-to-multipoint master-slave mesh, symmetrical mesh

: yes

: command/response/event messages

: optional, 3 available modes

: duty cycle individually configurable for each node

: end-to-end ACK implied by response to command or by explicit ACK request

Integrated Application Support : default configuration includes two 12-bit ADC channels, 3 -digital input channels with a 24-bit pulse-count option on 1 input, 2 digital output channels, all reconfigurable

DM2200-916VM Module Characteristic Specification [Copyright 2006 - 2007 CIRRONET]

FCC Certification

Operating Frequency

Modulation

Receiver Sensitivity

Receive Mode Current

Peak Transmitter Output Power

Peak Transmitter Output Current

Sleep Mode Current

Analog Input Measurement

Regulated Power Supply Input Range

Operating Ambient Temperature
: 15.247 Module Certification

: $916.5 \mathrm{MHz}$

: OOK on BPSK Spreading Code (OOK: On/Off Keys and BPSK: Binary phase-shift keying (BPSK)

: 10-3 BER @ -102 dBm typical

: 5.5-mA typical

: 10-dBm typical

: 32-mA typical

: $68 \mu$ A typical (RTC \& interrupts active)

: 12-bit, 3 selectable reference voltages

: 3.3 to $14-\mathrm{Vdc}$

: -40 to $85 \mathrm{C}$

Available Hardware Interfaces on DM2200-916VMs [Copyright 2006 - 2007 CIRRONET]

This generic module provides the following application hardware interfaces:

1. Serial port for data communications

2. 12-bit ADC for analog signal measurements

3. Digital I/O capabilities

4. Analog input capability

There are seven general purpose I/O ports available for application interfacing, GP1 - GP7, and three auxiliary digital ports, CFG0 - CFG2. Port GP0 is dedicated to receive signal strength 
indicator (RSSI) readings, and should always be defined as an analog input. Ports GP1 through GP7 can be individually configured as analog inputs, digital inputs or digital outputs. However, GP1 and GP2 have special capabilities when configured as digital inputs, and GP4 and GP5 have special capabilities when configured as digital outputs.

\section{Setting up a Network Using DM2200-916VM Radio Module ${ }^{3}$}

DM2200-916VM Radio Modules can be arranged in four different network topologies. The one we used is the symmetrical, multi-hop mesh network topology. This supports communications between any two nodes in the network. Traffic between two field nodes will not be routed through the master node unless the path through the master node is the most efficient path. There are four DM2200 modules available for this project. The modules are configured as a systematic multi-hop mesh network topology having three field node and one master node. One field node was placed on the roof that was connected to wind speed sensor and temperature sensor. One DM2200 module was configured as master node and connected to a computer via RS232. Two other DM2200 modules were placed at different floor locations in the building with only temperature sensors.

\section{There are several steps to setting up a network. They are as follows: ${ }^{3}$}

1. Installation of the utility/application software tools on to a computer.

2. Making hardware connection of master node module to the computer via RS232 or USB port.

3. Designating and configuring master node also refer to as base station by sending it a sequence of configuration command as follows:
a) A command is sent to the node to retrieve its current node ID, electronic serial number (ESN), and the master ID.
b) A command is then sent updating the node ID. This ID must be unique within the network.
c) Next, a command is sent, using the new node ID, to set the value of several connectivity beacon parameters. These will be passed on to any node being bound to the master node's network.
d) A command is then sent to set the network ID. Only messages carrying this node ID will be handled by the network. This allows overlapping networks to operate independently.
e) Next, a command is sent to set the master node beacon interval, typically in the range of 10 to 60 seconds.
f) Finally, a command is sent to set the operating mode of the node as a master node.

Once all nodes are bound and data-flow to the computer station was streamlined, we left the system ON for almost 24 hours. A part of the data collected by the system is graphical presented in Figure 3. Analyses of the collected data are presented under discussion. 


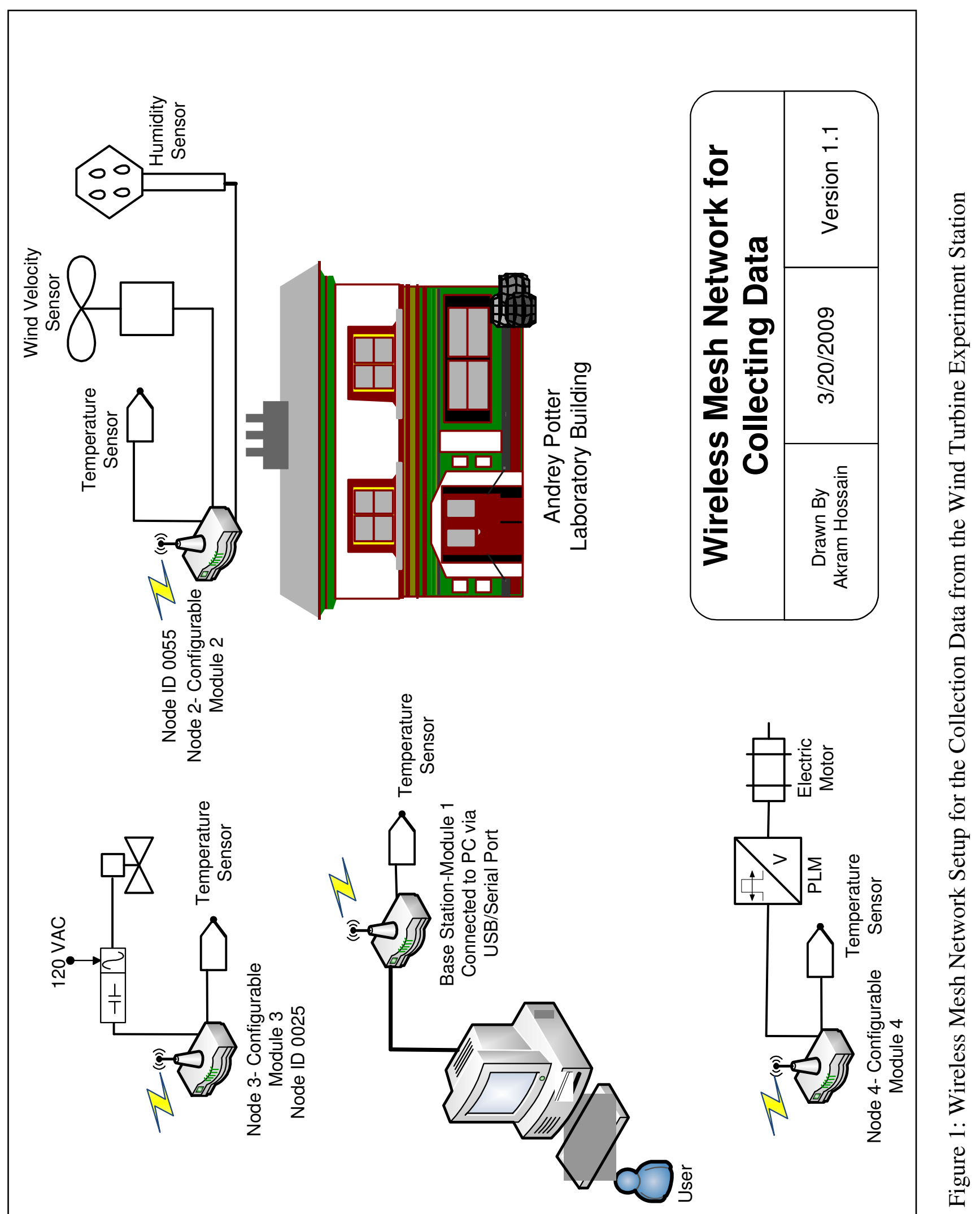

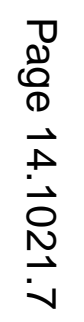




\section{Network Operation and Collection of Data from Field Nodes ${ }^{3}$}

Beacons sent by the master are monitored by each field node to optimize and/or recover routing to the master node, to assess path's robustness, and to synchronize power management cycles. The beacon period is a configurable parameter, with a default value of 29 seconds. Setting the beacon period longer helps improve power management and extend battery life, with the tradeoff of slower overall network dynamics. Setting the beacon period shorter improves the dynamics of a network, but lessens the effectiveness of power management. Data collecting setup that we have does not need to be speedy rather nodes' power management is more important for the conservation of battery life.

The beacon interval must be coordinated with the maximum number of hops required by the network. As a general rule, $250 \mathrm{~ms}$ should be allocated for each hop in a DM2200 network. The beacon period should be no less than 10 times the propagation time through the maximum number of hops in the network. For example, if a network uses a maximum for four hops, the maximum route propagation time is one second. The beacon period should then be at least 10 seconds. Since our data collecting network needed only two hops, beacon period for our network should at least be 5 second. Since the speed was not a big issue for our wind and temperature data collecting system, we varied the beacon period from 5 to 30 seconds.

Master node sends the command to read the each configured port of each bound field node. Each field node then transmits the data to the master node. We are collecting wind speed and temperature data from our rooftop wind turbine station having node ID 0055 and temperature only data from two other nodes having node ID as 0035 and 0025. Data collected from three nodes are received by the master node in turn stored in an MS Excel formatted sheet located in the host computer. Graphical User Interface (GUI) reads the computer clock and arranges the collected data in a date-time wise format in an excel sheet.

Each field node has the analog to digital converter input, digital input, and digital output capability. Each field node also has two $\mathrm{C}$-form relay which can be operate by making high/low configured digital port. Each port of the each node is reconfigurable and can be configured as the digital input, digital output and analog to digital input port as per the requirement.

The following dialog window appears when the application is launched. When the com port is selected, master node sends the master beacons to all field nodes to get connected with master node and at the same time application code (in Visual Basic) generate the excel sheet using the MS office program.
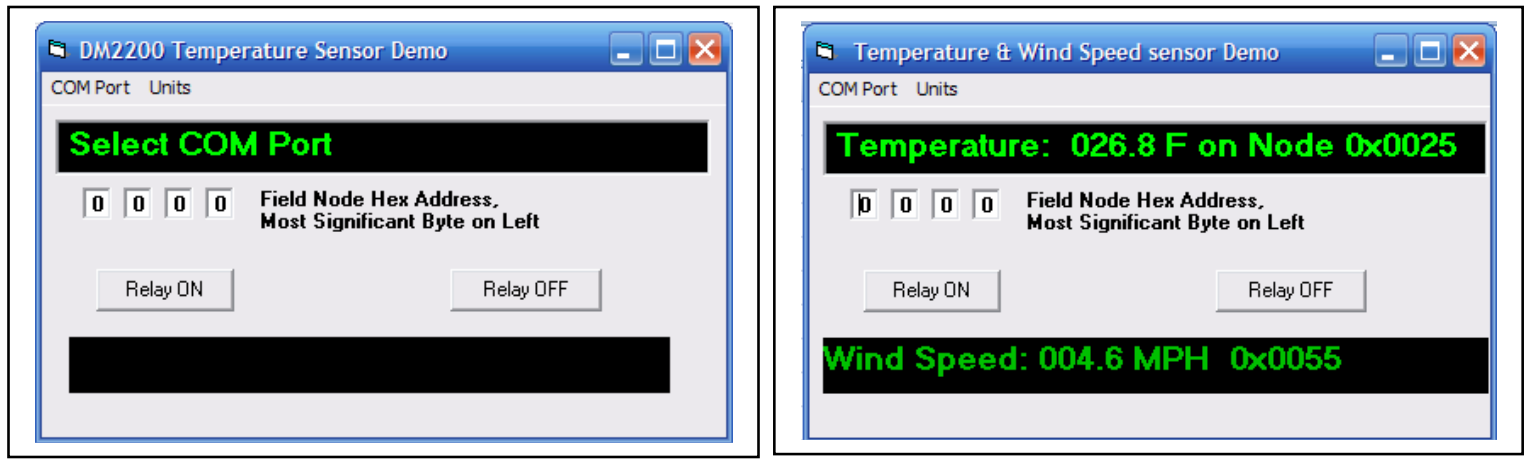
Generally COM 1 is selected if connected via RS 232, so we select COM Port 1 . We have the choice to select the unit (Degree F or $\mathrm{C}$ ) for the temperature reading and wind speed is measured in MPH. Field node's hex address code is provided, if reading from a specific node is desired. The above window shows actual temperature and windspeed reading from the rooftop wind turbine experimnt station for which the node ID is 0055.

However, if individual nodes reading are not desired the master node sends read command to all nodes and scans all ports of each node in a sequential order, collects the data from each node, and writes them in to a excel sheet. Figure 2 shows part of the excel data sheet having actual temperature and wind speed data collected from various nodes. The node data are displayed in excel sheet by time, date, and node number. This display on the host computer will update every 4 seconds. Since only one port data of a node is transferred at one time to the base node and in turn to computer excel sheet, ports that are located at distance node takes longer time to travel to to the base station. Although request for data from the master node is done at a specific interval, time it takes for the data to travel to master node depends on the distance and the number of hops the data has to make to get to the base station (master node). Note that one of the few limitation of a wireless mesh network is that it can hold onle a single communication at a given time.

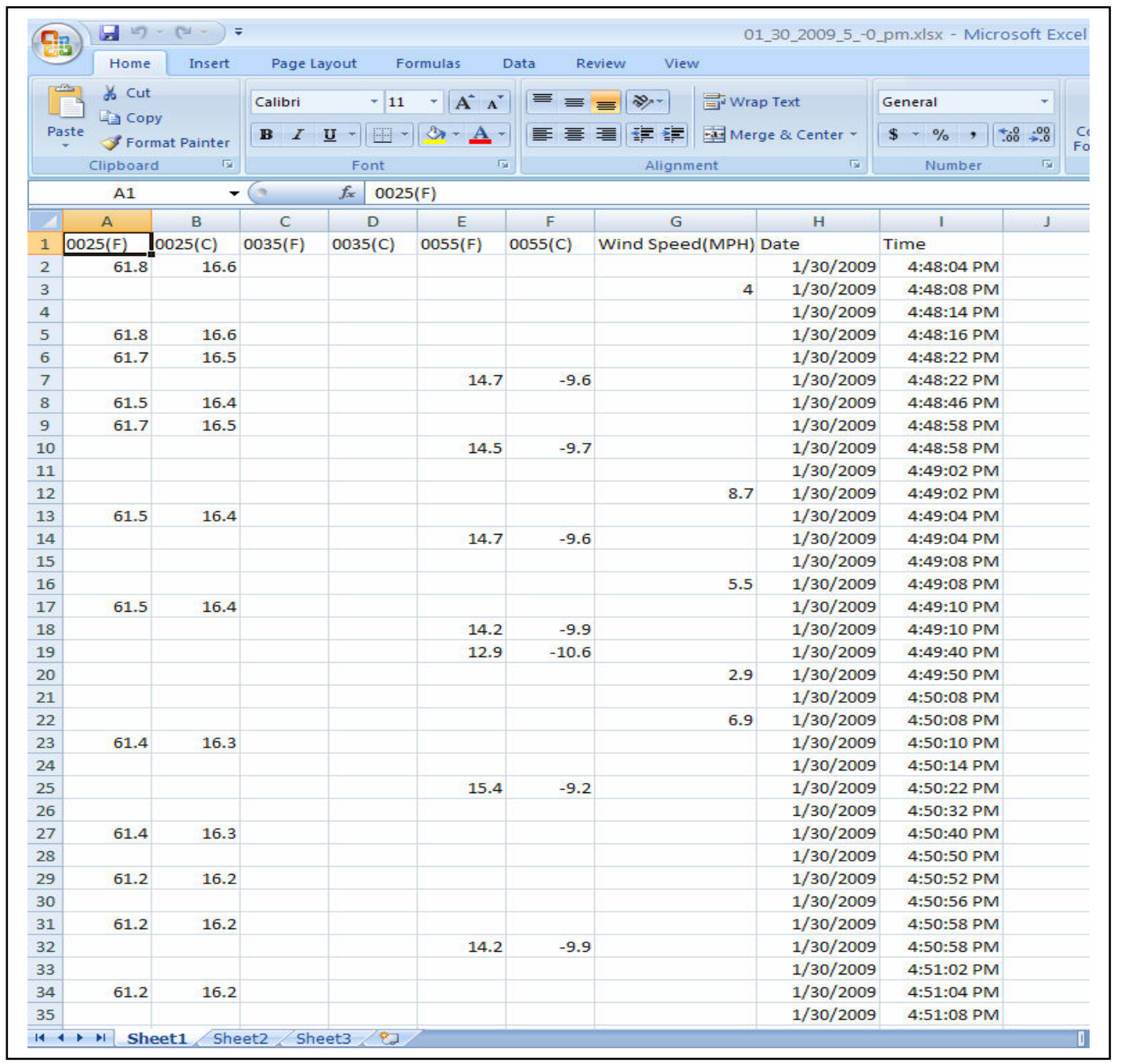

Figure 2: Part of the Excel Data Sheet ${ }^{4}$ 
The following two figures shows wind speed transducer and temperature sensor at the rooftop location. ${ }^{4}$
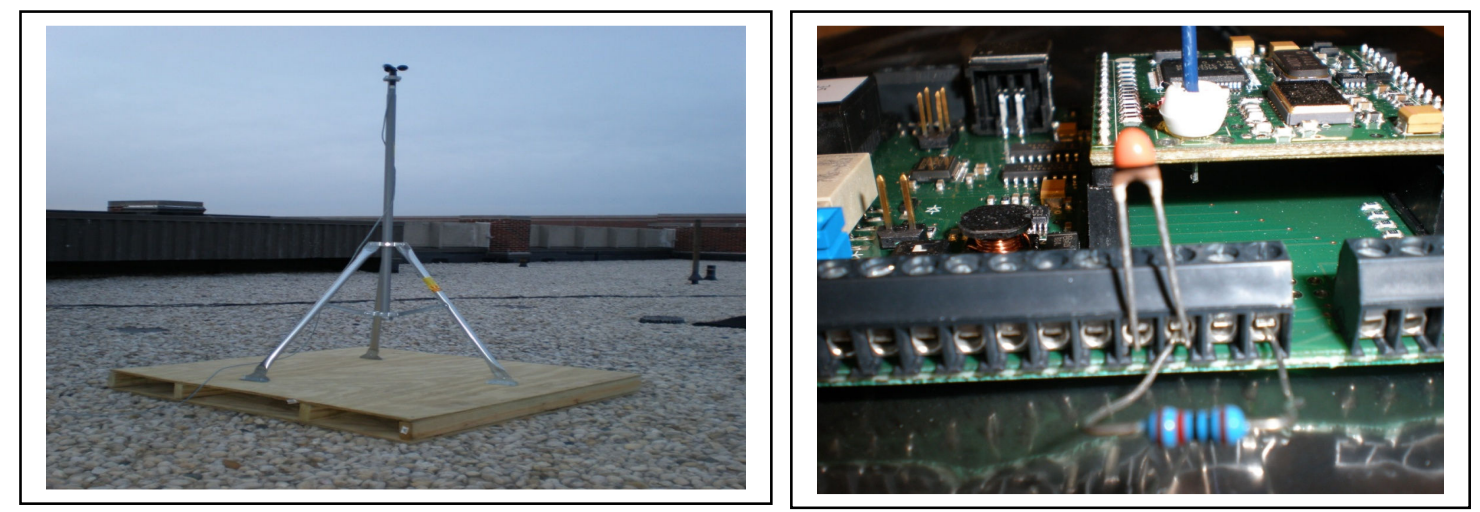

\section{Discussion}

Data collection started at 4:48:04 PM on January 30, 2009 and ended at 10:29:13 PM on January 31, 2009. A part of the data is presented in the form of a graph shown in Figure 3 below. Inside temperature was slowly decreased to $15^{\circ} \mathrm{C}$ and stabilized. Outside temperature declined from $-10^{\circ} \mathrm{C}$ to $-12^{\circ} \mathrm{C}$ over a period of about two hours. Wind speed was between $0-\mathrm{MPH}$ to $12-\mathrm{MPH}$. Over a 24 hour period mentioned above we found an average outside temperature of $14.87^{\circ} \mathrm{C}$ and an average temperature of $29.65^{\circ} \mathrm{C}$ inside the Potter building staircase. We also found an average wind speed was 5.4-MPH at the roof of Potter building that is about $60 \mathrm{ft}$ high from the ground level.

In the future we plan to expand our wireless mesh network to rooftops of other buildings within the campus and collect temperature, wind speed, wind direction, and humidity information. We plan to use these data for a feasibility study of wind turbines for harvesting electrical energy from wind.

\section{Conclusion}

Reconfiguring, hopping capability, inherent redundency, power management, robust network communication protocol through monitoring, analog inputs and outputs, digital outputs, and encryption for payload security all these features are very usful for our applications. In case of changing communication path environment, it is only necessary to place a repeater node in between the two module that has weak signal. Since the radios do not have to be large like a single hop system, cost of each module is considerably less and thus easily replacable. Hopping 
and easy of node mobility features makes it very convenient for our kind of distributed data collecting locations. Each field node periodically sends status maessage to master node to track connectivity between master node and field nodes. This enables host computer to maintain robustness of the network. Strength of signal path between mster node and field nodes are displayed by LEDs located on the master module and on individual modules. Four configurable mode of power management cycle is also very useful for our application. This allows us to have lasting battery life for our field modules that runs on battery in most locations.

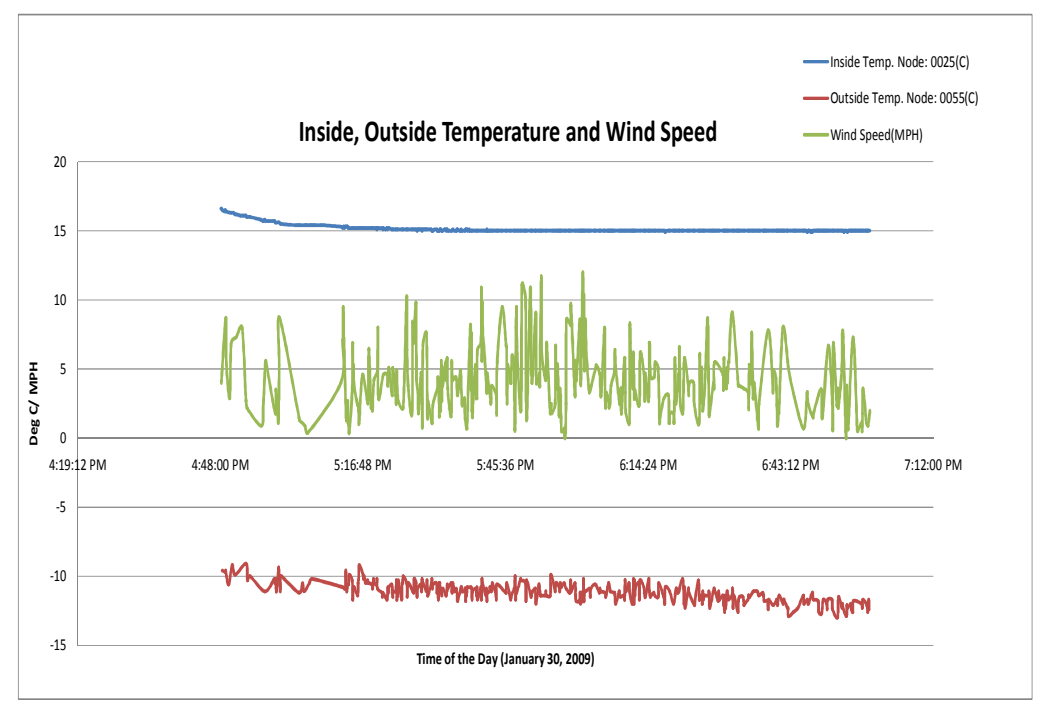

Figure 3: A Graphical Representation of the Collected Data

Omnex single-hop transcever system with four digital I/O and two analog I/O cost about $\$ 2000.00$ compared to this unit (DM2200) is only about $\$ 500.00$. In the past we have used Omnex wireless transceiver system for motor control and other similar application having lots of industrial noises.

Data integrity was not measured. However, claim was made for the following reasons: 1) The VersaMESH firmware includes a robust mesh networking protocol. 2) Multiple communication path makes the network system adoptive to sudden changes in path behavior and node hardware failure. 3) Acknowledgment mechanism in response to command. 4) Receive signal strength indicator (RSSI). 5) Periodic monitoring and transmiting of status message regarding conectivity 
between master node and each field node. These above features of the system ensure data integrity.

Data security is ensured by the following features of the network mesh: 1) Electronic serial number (ESN) and unique node ID for each field node and master node. This allows overlapping networks to work independently 2) Two payload encryption modes and three selectable 128-bit encription keys. 3) The network firmware is a highly customized version of TARP (tiny ad-hoc routing protocol).

Sensors are interfaced with the computer. However, currently available hardware only supports digital outputs and does not have any analog output (such as 4-20mA or 0-10V).

\section{References}

[1]. Yan Zhang, Wireless Mesh Networking, Architectures, Protocols and Standards, pp. 116-117.

[2]. Thomas Krag and Sebastian Buettrich (01/22/2004), from http://www.oreillynet.com/pub/a/wireless/2004/01/22/wirelessmesh.html

[3]. DM2200 Wireless Mesh Network from RFM website-www.rfm.com and www.cirronet.com

[4]. Vijay Mahajan, Wireless Mesh Network for Bidirectional Data Communication, A directed project for partial fulfillment of the Master of Science in Technology, Purdue University Calumet. Hammond, IN, Fall 2008.

[5]. Wi-Fi is a trademark of the Wi-Fi Alliance, founded in 1999 as WECA, Wireless Ethernet Compatibility Alliance. The organization comprises more than 300 companies, whose products are certified by the Wi-Fi Alliance, based on the IEEE 802.11. 